\title{
Distributed Generation with Voltage Control Capability in the Low Voltage Network
}

\author{
F. Sulla ${ }^{1}$ J. Björnstedt ${ }^{1}$ and O. Samuelsson ${ }^{1}$ \\ ${ }^{1}$ Department of Industrial Electrical Engineering and Automation \\ LTH, Lund University \\ IEA, LTH, PO BOX 118, SE-22100 Lund (Sweden) \\ Phone/Fax number: 0046 (0)46 22201 33, e-mail: Francesco.Sulla@iea.Ith.se, Johan.Bjornstedt@iea.Ith.se
}

\begin{abstract}
Small-scale distributed generators connected to the low voltage network are generally operated with unity power factor. However, controlling their reactive power injection would have an impact on the voltage at their connection point, if the network is sufficiently weak and inductive. The purpose of this paper is to investigate how a small-scale distributed generator connected to a low voltage network through a power electronic converter can be controlled to improve the voltage quality at its connection point.
\end{abstract}

The possibility to control the voltage at a point in the low voltage network by reactive power injection is limited by two factors, the small rating of the distributed generator compared to the short-circuit power at its connection point and the resistive nature of the low voltage cables. Here, a scheme for inductively decoupling the distributed generator connection point from the network is proposed and evaluated. For this purpose, an inductance is placed between the network and the generator, allowing voltage regulation by reactive power injection.

Simulation results and lab measurements show that the proposed scheme improves the voltage quality at the microturbine connection point.

\section{Key words}

Distributed Generation, Voltage Control, Inductive Decoupling, Low Voltage Network, Power Electronic Converter

\section{Introduction}

The amount of installed small-scale distributed generation has been growing in the last years. In many countries, government policies have favored the installation of new small-scale distributed generation. Distributed generation has brought up the possibility of making a more efficient use of fuel, even at the domestic level, as is the case with micro-CHP, or utilizing renewable energy sources available on-site, as in the case of solar power. Distributed generation could offer ancillary services as well. One such application is the possibility to operate the distributed generator in island mode during grid power outages. Such application could increase the power supply reliability. Methods for managing distributed generation units and exploiting their capabilities can be termed Active Network Management [1]. One issue where distributed generation plays a role is voltage regulation: Distributed generation affects voltage but can also be used to control voltage. Today distributed generation is primarily operated at unity power factor. This is to avoid voltage regulation problems in the low voltage network. Distributed generation interfaced to the network through power electronic converters however has the potential for voltage regulation.

This paper focuses on small-scale distributed generation for domestic use connected to the low voltage network. It aims at showing that a small $7 \mathrm{~kW}$ rated microturbine connected to the grid through a power electronic converter can be used to improve the voltage quality at its connection point, by controlling the converter reactive power. Because of the low rated current of the converter as compared to the short-circuit current in low voltage networks, the possibility of influencing the voltage by reactive power injection is limited. Moreover, the impedance in low voltage networks may be mainly resistive, thus limiting even more the possibility of voltage regulation by reactive power injection. To influence the voltage at the converter connection point by reactive power injection, the short-circuit power at that point must be purposely lowered and the network impedance must be mainly inductive.

One way of achieving both these conditions is to connect a properly dimensioned inductance between the converter and the network [2,3]. The inductance will here be referred to as "decoupling inductance". This allows voltage regulation at the microturbine side of the decoupling inductance, where sensitive loads can be connected. In reference [2], simulations during grid faults and interruptions and lab tests on a single-phase system 
are reported. Reference [3] uses a variable inductance. Here, a fixed inductance is used and the focus is on voltage disturbances in the grid during non-faulted conditions. A three-phase system is considered both in the simulations and in the lab tests. Connecting a singlephase converter to the grid via the decoupling inductance may not reveal one issue arising when a three-phase converter is connected instead. In this last case, a Dyn transformer must be used to feed single-phase loads, because of the fact that the phase-voltage on the converter side floats with respect to the grid neutral due to the interposed decoupling inductance and the PWM switching in the converter.

The quality of the voltage at the microturbine connection point depends on the short-circuit power of the network at that point: A nearby motor start can cause significant voltage dips in weak low voltage networks, while having no practical influence on the voltage in stronger low voltage networks. Other events such as load variations in the low and medium voltage network, capacitor switching or transformer tap changing in the medium voltage network may also influence the voltage at the microturbine connection point. The capability of the microturbine converter unit of regulating the voltage during these events has been investigated through simulations in MATLAB SimPowerSystems. The model is though to be integrated in the future in a real-time simulation environment, based on dSPACE, used as a real-time simulator for power electronics, and RTDS, used as a real-time simulator for the electricity network [1]. A converter prototype, to be used with the microturbine, has been developed and tested in the lab. Results from both simulations and lab measurements are presented.

Section 2 gives a brief theoretical basis for the inductive decoupling. Section 3 describes the microturbine and the power electronic converter. Section 4 describes the considered low voltage network model. Section 5 presents the results of the simulations. In Section 6, the measurements of the converter prototype lab tests are presented. Conclusions are presented in Section 7.

\section{Inductive Decoupling}

The use of a decoupling inductance to control the voltage at the converter connection point is justified by two reasons. First, in low voltage networks with long cables the network impedance may have a predominant resistive character. This limits the voltage change due to reactive power injection. This fact can be briefly explained by looking at the expressions for active and reactive power transfer across a resistive impedance.

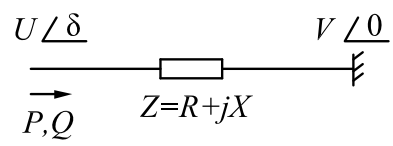

Fig. 1. Active and reactive power in feed to the grid.
With reference to Fig. 1, in the case of a resistive impedance the following holds:

$$
\begin{aligned}
& P=\frac{U^{2}-U V \cos \delta}{R} \\
& Q=-\frac{U V \sin \delta}{R}
\end{aligned}
$$

The active power is then dependent mainly on the voltage magnitude difference between the connection point and the grid voltage. This also means that the connection point voltage can be controlled by active power injection. To control this voltage by reactive power injection, the network impedance must be inductive, and this is here achieved with the decoupling inductance

The second reason to use a decoupling inductance is that the rated reactive power output of the converter is too small to significantly influence the voltage, even in weak low voltage networks. The decoupling inductance has therefore the role of weakening the network at the microturbine connection point in order to permit a predefined maximum voltage change when varying the microturbine reactive power from zero to rated value. In this case the converter has been dimensioned to supply 7 $\mathrm{kW}$ active power and 7 kvar reactive power simultaneously. The maximum available reactive current amounts to $10.1 \mathrm{~A}$ at $400 \mathrm{~V}$. It has been considered that this reactive current limit is valid independently of the amount of the delivered active power. The value of the decoupling inductance has been chosen to obtain a voltage variation over the inductance of approximately $\pm 10 \%$ of the nominal phase-voltage. A decoupling inductance of $7.3 \mathrm{mH}$ allows, with rated reactive power injection, a change of $\pm 23 \mathrm{~V}$ in the phase-voltage over the decoupling inductance.

\section{Description of microturbine model}

The converter prototype to interface the microturbine to the network has been built and tested in the lab. A simulation model of the microturbine converter has been implemented in MATLAB SimPowerSystems. The microturbine is rated $7 \mathrm{~kW}$ and feeds the active power into the DC-link capacitors via a diode rectifier and a step-up converter.

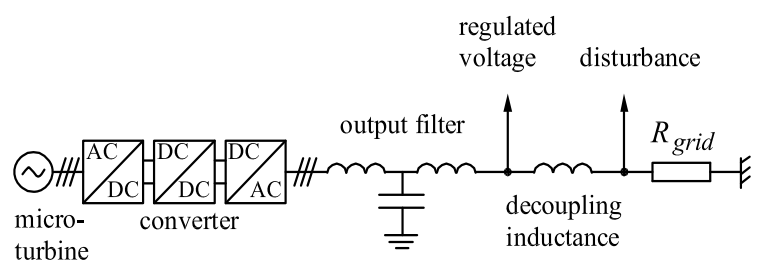

Fig. 2. Microturbine and converter connected to the grid through a filter and a decoupling inductance.

The DC voltage is then converted to a three-phase AC voltage with a power electronic converter. To allow voltage regulation by reactive power control the 
converter must be uprated as compared to the microturbine generator rating. A scheme of the microturbine, the power electronic converter with LCLfilter and the decoupling inductance is shown in Fig. 2.

\section{A. Microturbine and step-up converter}

The generated active power can be assumed constant in this analysis due to the high time-constant of the thermal system compared to the analyzed fast network transients. Therefore, in all simulations and lab tests the active power has been set to a constant value. The current delivered by the microturbine generator is rectified and fed into a step-up converter. The rectified output voltage of the generator is assumed constant and equal to $350 \mathrm{~V}$. In reality, this voltage will not be constant due to the generator internal impedance. The step-up converter is used to increase the generator voltage to the DC-link voltage of $750 \mathrm{~V}$.

\section{B. Three-phase converter}

A three-phase converter interfaces the microturbine to the network, as shown in Fig. 3. The converter output stage must include a passive filter to fulfill the requirements on current harmonic feed into the power grid.

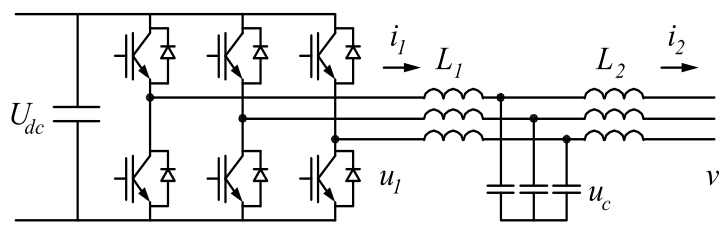

Fig. 3. Three-phase converter

The purpose of the filter is to damp the harmonics introduced by the PWM switching. The standard IEC 1000-3-4 states that current harmonics above the $33^{\text {rd }}$ should be less than $0.6 \%$ of the nominal converter current. In this project an LCL filter has been considered, whose components have been chosen according to [4] and are reported in Table I.

Table I - Three-phase converter data

\begin{tabular}{cc}
\hline \hline Quantity & Value \\
\hline$L_{1}$ & $2.2 \mathrm{mH}$ \\
$R_{1}$ & $75 \mathrm{~m} \Omega$ \\
$C_{d c}$ & $10 \mu \mathrm{F}$ \\
$L_{2}$ & $1.15 \mathrm{mH}$ \\
$R_{2}$ & $66 \mathrm{~m} \Omega$ \\
$k_{i 2}$ & 1 \\
$k_{u c}$ & 0.2 \\
$k_{i 1}$ & 0.1 \\
$k_{p s}$ & 0.5 \\
\hline \hline
\end{tabular}

An LCL filter has some advantages over a common L filter [4], due to the fact that an LCL filter presents increased attenuation of high order harmonics while allowing the use of lower size inductances. However, when using an LCL filter, resonances may arise which must be dealt with [5]. The resonance problem is dealt with in the lab converter prototype, switched at $4950 \mathrm{~Hz}$, by using a notch filter designed to match the resonance frequency. The filter is applied on the reference voltage output by the LCL filter current controller. The frequency response from the converter output voltage, which contains the harmonics originating from the PWM switching, to the line current is shown in Fig. 4. The converter control system has been modeled in $d q$-coordinates and it includes an $\mathrm{AC}$ voltage regulator, a DC-link voltage regulator and a current controller, as shown in Fig. 5. The AC voltage regulator outputs the $q$-reference current to regulate the voltage $v$ after the LCL filter at its reference value. The DC-link voltage regulator outputs the $d$-reference current to keep the DClink voltage at nominal value. The $d$-current determines the amount of active power delivered to the network. AC and DC voltage regulators are both implemented as PI-controllers. The current controller is made up of three cascaded stages.

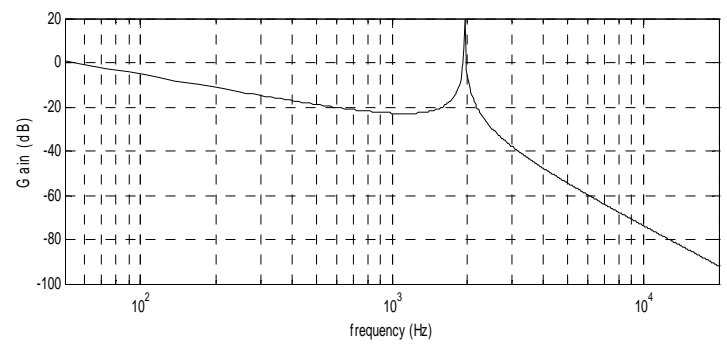

Fig. 4. Frequency response from converter output voltage $u_{1}$ to line current $i_{2}$.

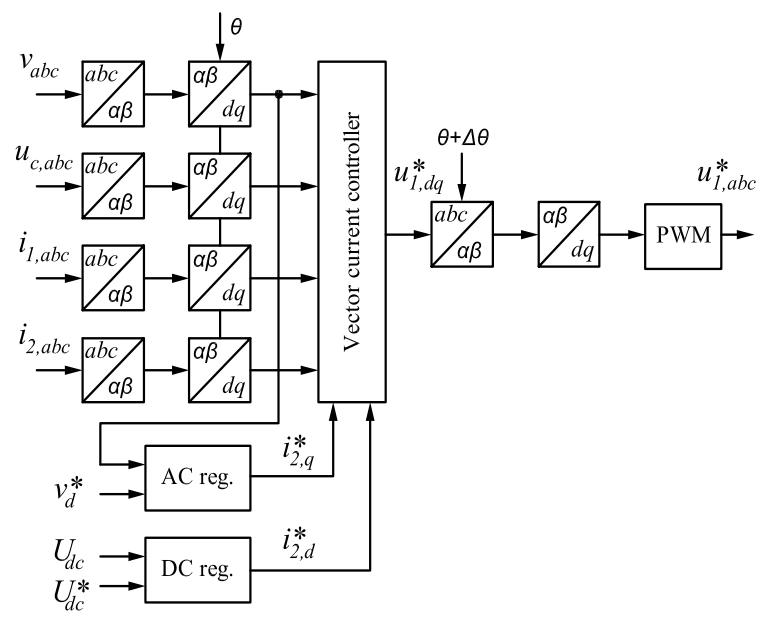

Fig. 5. Converter control scheme.

The first stage controls the current in the outer LCL filter inductor $L_{2}$, the middle stage controls the filter capacitor voltage while the third stage controls the current of the inner filter inductor $L_{1}$. The inputs to the current controller are the voltages at the converter side of the decoupling inductance, the filter capacitor voltage and the currents in the two filter inductances. A Smith predictor can be used to predict the current in the inner inductor $L_{1}$ one sample ahead. This is to compensate for the one sample delay introduced by the use of a DSP in the converter prototype. The converter control is 


$$
\begin{aligned}
& u_{c}^{*}(k)=k_{i 2} \frac{L_{2}}{T_{s}}\left[\left(i_{2}^{*}(k)-i_{2}(k)\right)+\sum \Delta i_{2}(k)\right]+\left(R_{2}+j \omega L_{2}\right) i_{2}(k)+v(k) \\
& i_{1}^{*}(k)=k_{u c} \frac{C}{T_{s}}\left(u_{c}^{*}(k)-u_{c}(k)\right)+j \omega C u_{c}+i_{2}^{*}(k) \\
& u_{1}^{*}(k)=k_{i 1} \frac{L_{1}}{T_{s}}\left[i_{1}^{*}(k)-\left(i_{1}(k)+i_{\text {smith }}(k)\right)\right]+\left(R_{1}+j \omega L_{1}\right) i_{1}(k)+u_{c}(k) \\
& \sum \Delta i_{2}(k+1)=\frac{T_{s}}{T_{i}}\left(i_{2}^{*}(k)-i_{2}(k)\right)+\sum \Delta i_{2}(k) \\
& i_{\text {smith }}(k+1)=\left(1-\frac{R_{1} T_{s}}{L_{1}}-j \omega T_{s}\right) i_{\text {smith }}(k)+\frac{T_{s}}{L_{1}}\left(u_{1}^{*}(k)-u_{c}(k)\right)+k_{p s}\left(i_{1}(k)-i_{\text {smith }}(k)\right)
\end{aligned}
$$

implemented only for the positive sequence and this limits the performance of the controller in cases where appreciable unbalances in the network voltage arise. This could be partly compensated for by using a suitable control algorithm, but it has not been done here. The current controller is implemented as in [4] and it is shown in (2). In [4] the values of the parameters gains are chosen as $k_{i 1}=0.5, k_{u c}=0.25, k_{i 2}=1$. This choice of parameters resulted in a low damped oscillatory behavior of the controller when using a decoupling inductance, $L_{\text {dec }}=7 \mathrm{mH}$, in the simulations. To guarantee better stability margins, the gain $k_{i 1}$ must be further decreased. The chosen parameter values, reported in Table I, resulted in a stable and damped behavior of the converter in the simulations. In the lab tests, the converter control proved to be stable even with higher values of $k_{i 1}$.

\section{Description of Network Model}

Both a medium voltage and a low voltage network are included in the simulations. The medium voltage network is composed of one feeder, modeled as a pi-link. A low voltage network is tapped from it through a distribution transformer. The distribution transformer rating and the impedance to the microturbine converter unit connection point are changed in order to consider a strong and a weak connection point. In the strong network case, the short circuit current at the converter connection point is about $8 \mathrm{kA}$, while in the weak network case it is around $0.4 \mathrm{kA}$. A bank of switched 2.5 Mvar capacitors can be connected at the medium voltage network to simulate a change in the grid voltage. The values of the low voltage cable data used in the simulations for the weak network are reported in Table II. In the lab tests, the cable between the grid and the microturbine connection point has been modeled by $0.7 \Omega$ resistances as phase conductors and a $1 \Omega$ resistance as PEN conductor.

Table II - Data for low voltage weak network

\begin{tabular}{cc}
\hline \hline Quantity & Value \\
\hline$R_{\text {phase-weak }}$ & $0.5 \Omega$ \\
$L_{\text {phase-weak }}$ & $0.27 \mathrm{mH}$ \\
$R_{P E N \text {-weak }}$ & $0.4 \Omega$ \\
$L_{P E N \text {-weak }}$ & $0.12 \mathrm{mH}$ \\
\hline \hline
\end{tabular}

\section{Simulations}

The cases considered in the simulations include events on both the medium and the low voltage network. Threephase and single-phase motor starting in the low voltage network have been simulated. The three-phase induction motor is rated $2.5 \mathrm{~kW}$ and a standard model included in the simulation package was used. The motor is loaded with a torque proportional to the square of the motor speed.

The single-phase motor, rated $1 \mathrm{~kW}$, is simulated by playing back into the simulations the measured start-up current of a vacuum cleaner. Both motors are connected on the grid side of the decoupling inductance, i.e. near the converter connection point. At this same point, a $3 \mathrm{~kW}$ resistive load is connected during all simulations. The simulated events on the medium voltage network include the insertion and the successive disconnection of the 2.5 Mvar capacitor bank. Both these events cause a voltage variation in the system and at the microturbine connection point.

All the events have been simulated with the microturbine and the decoupling inductance connected to both a strong and a weak low voltage network. As it may be expected, motor start-up causes almost no voltage dip in the strong network case. Events on the medium voltage network, like the capacitor switching in the simulations, on the other hand affect the voltage in both a weak and a strong low voltage network. Thus the microturbine is effective in improving voltage quality especially when connected to a weak network, but it may also counteract voltage changes coming all the way through the medium voltage network. Three different values of active power delivered by the microturbine have been considered: $1,3.5$ and 7 $\mathrm{kW}$.

When the microturbine is connected to a weak low voltage network, it experiences higher voltage variations compared to when connected to a stronger network. Therefore, the results from the simulations of the microturbine connected to a weak network are of interest in judging its voltage control performance. In the following figures, the voltages on the grid and converter side of the decoupling inductance are compared. The voltages are expressed in pu of their values before the occurrence of the simulated event. It must be noted that 
these values are not the same when expressed in volts. In fact, the voltage on the microturbine side is regulated to nominal value, while the grid side voltage is at a lower value. The three-phase $2.5 \mathrm{~kW}$ motor start causes a voltage dip of about $0.06 \mathrm{pu}$ on the grid side of the decoupling inductance. At the microturbine terminals, however the voltage dip is less severe and of shorter duration, see Fig. 6. The microturbine voltage controller mitigates the dip and reestablishes nominal voltage also after the starting process is over, while the voltage on the grid side of the decoupling inductance decreases as compared to its value before the motor connection. The same is valid for the single-phase motor start shown in Fig. 7. However in this case, because of a negative sequence voltage appearing at the microturbine terminals, the voltage in the other two phases increases slightly above nominal value.

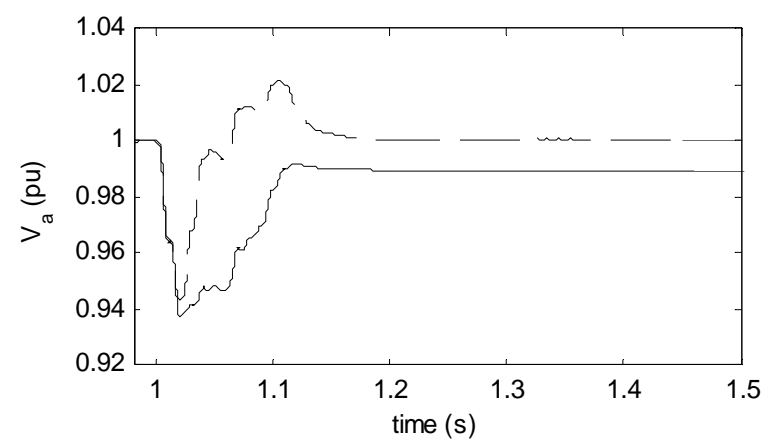

Fig. 6. RMS voltage in phase-a on the grid side (solid) and at the converter side (dashed) of the decoupling inductance. $2.5 \mathrm{~kW} 3$-phase motor start.

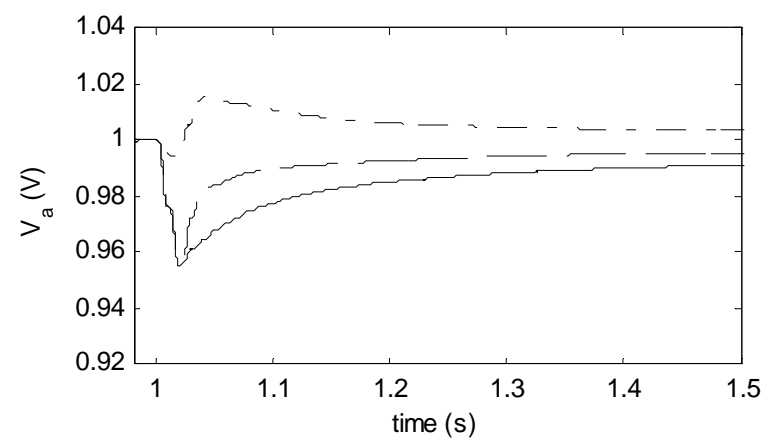

Fig. 7. RMS voltage in phase-a on the grid side (solid) and on phase-a (dashed) and phase-b (dashed-dotted) at the converter side of the decoupling inductance. $1 \mathrm{~kW}$ single-phase motor start.

The current controller has been implemented by assuming that symmetrical conditions are present in the network. This causes a ripple in the output power and the DC-link voltage of the microturbine during unbalanced conditions [6].

A voltage change originating from the medium voltage network, and therefore transmitted over the entire low voltage network, is counteracted equally well by the microturbine controller. The voltage change originated by the insertion - disconnection sequence of a 2.5 Mvar capacitor bank in the medium voltage is shown in Fig. 8.
The microturbine controller quickly regulates the output reactive power to get nominal voltage at its terminals. Simulations show that the amount of active power delivered by the microturbine before and during the disturbance does not affect its voltage regulation capability.

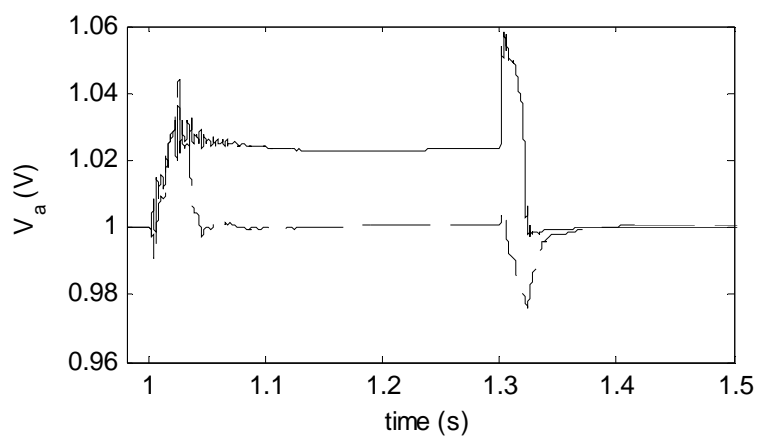

Fig. 8. RMS voltage in phase-a on the grid side (solid) and at the converter side (dashed) of the decoupling inductance. 2.5 Mvar capacitor insertition - disconnection in the medium voltage network.

\section{Lab Tests}

A converter prototype has been set-up in the lab and its control has been realized with the aid of a Digital Signal Processor. The microturbine has been replaced by a DC generator, driven by a grid-connected induction motor. The step-up converter strives to control the output current from the DC generator to a constant pre-set value. The converter has been connected to the grid via a decoupling inductance. Resistances, as described in Section 4 have also been added in between the decoupling inductances and the strong grid, in order to reproduce the conditions for a weak network.

Single-phase loads at the converter side of the decoupling inductance are connected on the secondary side of a Dyn transformer. In the tests, the y-side was at a lower voltage level than the D-side, but in reality a 1:1 ratio transformer should be used to feed $230 \mathrm{~V}$ single-phase loads. The need of a Dyn transformer for connecting single-phase loads is due to the fact that the phase-voltage on the converter side floats with respect to the grid neutral due to the interposed decoupling inductance and the PWM switching in the converter. Connecting a single-phase load directly between one converter phase and the grid neutral would therefore result in high ripple load current. The measurements from the starting of a $0.37 \mathrm{~kW}$ threephase fan motor are shown in Fig. 9. Measurements of the voltage over a resistive load connected on the $y$-side of the Dyn transformer, during the starting of a $1 \mathrm{~kW}$ single-phase motor, are reported in Fig. 10. In both cases, the motors have been connected on the grid side of the decoupling inductance.

It can be seen that the converter control action has a very positive effect on the voltage. This is true even when a small-rated $1 \mathrm{~kW}$ single-phase motor, as a common vacuum cleaner like the one used in this test, is started in 
a weak low voltage network. The converter control efficiently mitigates the voltage dip. As already said in

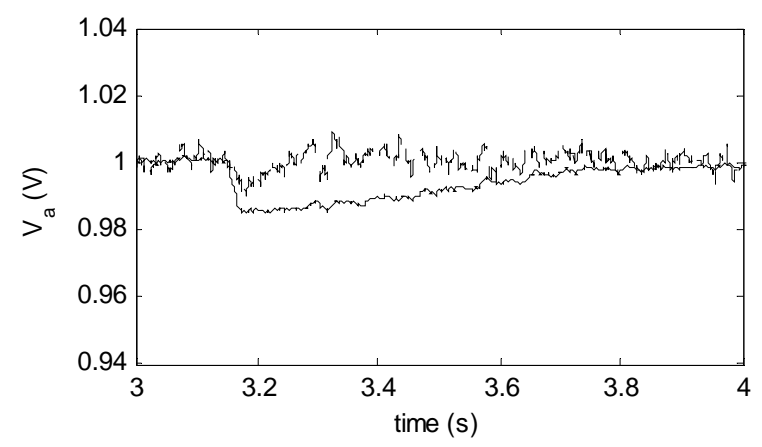

Fig. 9. Measured RMS voltage in phase-a on the grid side (solid) and on phase-a (dashed) at the converter side of the decoupling inductance. $0.37 \mathrm{~kW}$ three-phase motor start.

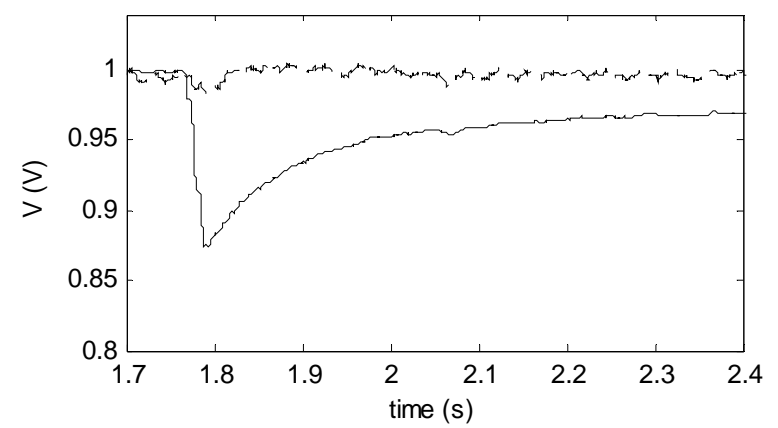

Fig. 10. Measured RMS voltage in phase-a on the grid side (solid) and on phase-a (dashed) over a resistive load connected on the $y$-side of the Dyn transformer at the converter terminals. $1 \mathrm{~kW}$ single-phase motor start.

Section 4 the implemented control does not handle unbalances and this resulted in phase-b and phase-c voltages at the converter terminals slightly above nominal values, during the single-phase motor start on phase-a.

\section{Conclusions}

A method for implementing voltage regulation using a small-scale microturbine interfaced to the grid through a power electronic converter has been implemented. Thanks to the presence of a decoupling inductance, the proposed voltage regulation scheme works independently of the strength at the grid connection point and of the nature, inductive or resistive, of the equivalent grid impedance at this point. In fact, also in very weak but mainly resistive networks, as the one considered here, a low rating converter is not able alone to influence the voltage without the use of a properly sized decoupling inductance.

Simulation results and lab measurements show that the proposed scheme improves the voltage quality at the converter connection point, effectively mitigating voltage dips appearing on the grid side of the decoupling inductance.

This is true when the disturbance causing the voltage dip is originating both on the low and medium voltage network. Loads connected between the converter and the decoupling inductance would therefore benefit from the converter voltage control scheme. The initial delivered active power of the microturbine shows to have no appreciable effects on its voltage control capability.

A drawback of this scheme is that single-phase loads at the converter side of the decoupling inductance must be connected through a Dyn transformer. This is because the phase-voltage on the converter side floats with respect to the grid neutral, due to the interposed decoupling inductance and the PWM switching in the converter. Connecting a single-phase load directly between one converter phase and the grid neutral would therefore result in high ripple load current. When unbalanced conditions arise, as in the cases of the single-phase motor start, a controller taking into consideration the unbalance in the network voltage would result in better performance.

\section{Acknowledgement}

The work presented in this paper has been conducted as part of the ADINE project, financially supported by the European Commission under contract no. TREN/07/FP6EN/S07.73164/038533.

\section{References}

[1] S. Repo, K. Mäki, O. Samuelsson, ADINE - EU demonstration project of active distribution network, CIRED seminar 2008: SmartGrids for Distribution, Frankfurt, 23-24 June 2008.

[2] Jahn J., Engler A., Inductive Decoupling of Low-Voltage Sub-Networks, 9th International Conference on Electric Power Quality and Utilization, Barcelona 2007.

[3] Morren J., Grid Support by Power Electronic Converters of Distributed Generation Units, PhD Thesis, Technical University of Delft, 2006.

[4] Bojrup M., Advanced Control of Active Filters in a Battery Charger Application, Licentiate Thesis, Department of Industrial Electrical Engineering and Automation, Lund University, 1999.

[5] Cobreces S., Bueno E., Rodriguez F., Huerta F., Rodriguez P., Influence Analysis of the Effects of an InductiveResistive Weak Grid over L and LCL Filter Current Hysteresis Controllers, IEEE European Conference on Power Electronic and Applications, 2007.

[6] Song H., Nam K., Dual Current Control Scheme for PWM Converter Under Unbalanced Input Voltage Conditions, IEEE Transactions on Industrial Electronic, Vol. 46, No. 5, October 1999. 